\title{
Falling Water with Key Particle and Envelope Surface for Virtual Liquid Manipulation Model
}

\author{
Shunsuke Miyashita \\ Nagoya Institute of Technology \\ Gokiso-cho, showa-ku, Nagoya 466-8555 Japan \\ miyasita@center.nitech.ac.jp
}

\author{
Kenji Funahashi \\ Nagoya Institute of Technology \\ Gokiso-cho, showa-ku, Nagoya 466-8555 Japan \\ Kenji@nitech.ac.jp
}

\section{Categories and Subject Descriptors}

H.5.1 [Information Interfaces and Presentation]: Multimedia Information Systems - Artificial, augmented, and virtual realities

\section{Author Keywords}

liquid manipulation model; key particle; envelope surface

\section{INTRODUCTION}

We have proposed a liquid manipulation model based on particle and volume, and a VR chemical laboratory system [1]. In the CG field, although researchers usually use particle method to simulate realistic fluid, it needs enormous computation time and usually needs parallel processing to realize real-time simulation [2]. One of our main goal is to provide the VR-learning system for the people, i.e., who have to stay in the hospital. Therefore we have already proposed an interactive model of virtual liquid like water which focus on user impression and real-time processing rather than exact behavior simulation. The model can make user feel realistic sensation from the water behavior manipulated by user's container. However free fall water was simulated and rendered with particles simply. The processing speed is influenced by a lot of particles, and CG quality is insufficient. In this paper, we propose an efficient and effective method of free fall water with key particles instead of conventional particles. The envelope surface is rendered around the key particles as a surface of the water. The new model can make more realistic sensation with higher processing speed.

\section{VOLUME \& PARTICLE BASED MODEL}

We usually manipulate water with container, and count it as "a cup of water." So we represent the water in our proposed model as following two conditions to simplify calculation; the stay condition which exists in a container and the free fall condition which flows from/to container. The former is treated with volume value, and the latter is treated as a simple particle system that particles do not interfere each other. Although water is not one object, we treat the water in a container as one object, and calculate water surface level from volume and its tilt. We have also developed the method to express wave, vortex and color diffusion on the surface. However the free fall condition water is represented

Copyright is held by the author/owner(s).

VRST'12, December 10-12, 2012, Toronto, Ontario, Canada.

ACM 978-1-4503-1469-5/12/12.
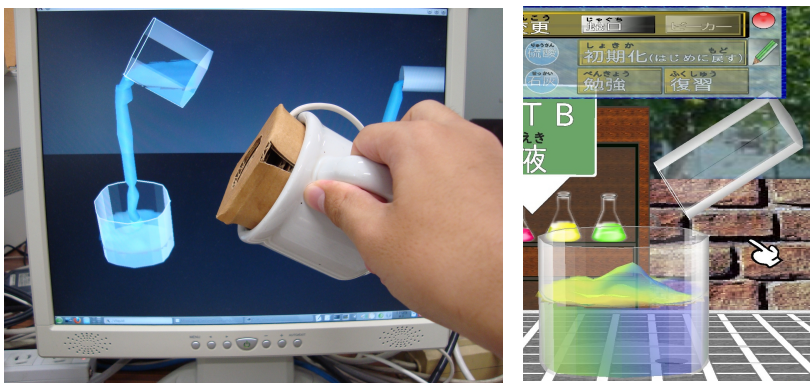

Figure 1: Experimental system

as just particles and rendered as simple square transparent polygons. Using this model, an interactive water manipulation is realized in real-time.

\section{KEY PARTICLE BASED MODEL}

In our new model, key particles are proposed to represent falling water behavior. They have their own location, volume and color parameters, and do not interfere each other as same as conventional particles. While water is flowing, key particles are generated at regular intervals, then each particle moves according to gravity and inertia. So the number of key particles is less than the conventional ones. When the flow is stopped, the particle segment is separated. Finally, envelope surfaces are rendered around each segment of the key particles. The surface is constructed as a frustum for each pair of adjoining key particles, base area is decided by the volume parameter of the particle.

\section{CONCLUSIONS}

We proposed the new model which represents falling liquid with key particles and an envelope surface as a part of the virtual liquid manipulation model. It can make more realistic sensation with higher speed (Fig. 1). As a future work, we should implement a VR chemical laboratory system on a mobile computer, and evaluate its validity from students. Acknowledgments: This work was supported in part by JSPS KAKENHI Grant Number 24501186.

\section{REFERENCES}

[1] Natsume, et al. The virtual chemical laboratory using particle and volume based liquid model. Proc. SCIS \& ISIS, 1354-1359, 2010.

[2] Losasso, et al. Two-way coupled SPH and particle level set fluid simulation. IEEE TVCG, 14(4):797-804, 2008. 